\title{
Fuzzy Logic-based Risk Assessment Framework to Evaluate Physiological Parameters
}

\author{
Edit Tóth-Laufer, Márta Takács, Imre J. Rudas \\ Óbuda University, Bécsi 96/b, H-1034 Budapest, Hungary \\ laufer.edit@bgk.uni-obuda.hu, takacs.marta@nik.uni-obuda.hu,rudas@uni- \\ obuda.hu
}

\begin{abstract}
Nowadays the patient monitoring systems are more and more widely used due to the advances in the technical background. These systems can improve the quality of life and by using them, several complications can be avoided in emergency situations. Nevertheless, the proper evaluation of the measured factors is a highly problematic task. The medicine uses a number of parameters, which are difficult to quantify, the boundaries of the examined risk factors cannot be given in a general form, the realistic result is only available from personalized thresholds. For these reasons, user-specific evaluation is required as much as possible, which uses individual characteristics, also the living conditions, habits and the medical recommendations taken into account during the risk calculation. In addition, the complex interactions between the input factors, which are often opaque, can be better handled in a patient-specific way based on the medical history of the patient or previous measurements. We developed a risk assessment framework to meet the above mentioned requirements. This system is flexible with a high degree of adaptive capacity. For the sake of ease of extensibility, and in order to achieve transparency, a generalized, modular system structure is implemented, which is joined with a database to specify the parameters of the configurable subsystems.
\end{abstract}

Keywords: risk assessment; fuzzy logic; relational database

\section{Introduction}

Risk management is again becoming more and more the focus of research, due to the economic crisis of recent years the increase in the number of terrorist activity; and extreme environmental and climatic conditions. Additionally, the novel recognizable and measurable risk factors in the field of medical diagnostics needed more efficient risk assessment schemes [1]. The large number of system parameters and the complexity of their context has led to the model's complexity, which should be handled adequately. Both quantitative and qualitative parameters are among the risk factors, therefore a method is needed during the evaluation, which can handle both types of inputs. Soft computing techniques are useful tools 
in risk management systems, which are full of uncertainty and subjectivity in the data and in the evaluation process [2]. In this paper, we primarily focus on the system, which examines the physiological parameters. These kinds of systems are integral parts of engineering, natural and social environments and they require generalized, multilevel risk management models.

Nowadays, the beneficial effects of regular exercise in prevention, rehabilitation, and active life are known to everyone. However, basic physical information, including chronic diseases, actual physical status, age, and many other sub-factors, should be considered, in order to keep the practiced sport safe and healthy rather than resulting in the situation becoming worse [3]. To avoid the serious consequences, the implementation of a real-time risk assessment system is justified in order to continuously control the current risk level.

Because of the problem of the aging population in the developed world these monitoring systems mainly focus on elderly people with chronic diseases [4]-[8]. They spend their days often alone and unattended, therefore an emergency frequently occurs unnoticed. However, with the use of monitoring systems, the patients' mortality can be significantly reduced and the course of disease can be better treated and controlled if the problem is detected in time [9]. The sounding of the alarm on time as well the arrival of the right person is of critical importance [10]. The monitoring during sport activity is of similar importance for the emergencies caused by unexpected problems, which can also be eliminated, as well as monitoring whether proper performance is achieved. Due to the large number of input factors and complex interactions between them, most systems examine only one measured parameter and some of its influential parameters [11], [12]. Usually the examined factors are physiological parameters [13], [14] but, in addition, motion detection can also be used [15], [16]. The aim of these systems may differ, they can also be applied as a teaching device in the fields of sports and rehabilitation [17], [18]. The fields of these kinds of systems are the same as the surveillance of the elderly (e.g. basic personal information, chronic diseases), but it should be supplemented by the sport habits (e.g. motion forms, intensity, frequency). Regardless of the measured parameters and the intended use, it can be concluded that these systems evaluate fix parameter combination, independently of the sport type and patient characteristics. In contrast, the novel risk assessment framework is able to work with patient- and sport-specifically defined parameter combinations.

In patient monitoring systems the boundaries of the risk factors, which are predominantly physiological parameters, cannot be given in a general form. There is no general definition of what is considered normal, increased, or perhaps an abnormal value [11]. Although absolute definitions are also used in medicine, the realistic result is only available from personalized thresholds. For these reasons user-specific evaluation is required as much as possible, using individual characteristics. Living conditions and medical recommendations are also taken into account during the risk calculation [19]. In addition to the complex 
interactions between the input factors, which are often opaque, can be provided more easily if the medical history of the patient is known or the previous measurements are available. In this case the interactions can also be given in a patient-specific way. The above mentioned requirements, as well as the large number of the considered risk factors, require the development of a robust, flexible risk management system with a high degree of adaptive capacity. For the sake of ease of extensibility, and in order to achieve transparency a generalized, modular system structure should be used, which is joined with a database to specify the parameters of the configurable subsystems [20]. We present our work about a risk assessment framework for physiological parameters, primarily during sport activity but it can be used in other areas (e.g. monitoring of elderly, home rehabilitation) with minor modifications in the database.

The rest of the paper is organized as follows: In Section 2, the basic structure of the fuzzy risk assessment model is described. Section 3 summarizes a hierarchical decision method (AHP). Section 4 and 5 present the evaluation process and the database design behind the framework. In Section 6, the implementation of the proposed risk assessment framework, the test environment and method are described. Finally, the conclusions are drawn.

\section{The Model Structure}

\subsection{The Fuzzy-based Risk Assessment Framework}

The risk assessment framework is applied in the authors' sport activity risk calculation model [3], which uses fuzzy logic based decision making in a hierarchical, clustered structure. The aim of the system is to calculate the risk level based on physiological and other personal parameters of the user to control the patient continuously. In the model there are some constant (e.g. sex) and quasiconstant parameters (e.g. age, occupation), which rarely change. The expert can design the personal profile of the patient based on these parameters. This profile, which is realised in a well-structured database, contains the types of activities, which should be monitored for the user, the name of the parameters, which should be measured during the monitoring assigned to the user, and their personal thresholds based on the medical recommendations. Neither the inputs, nor the parameters of the membership functions belonging to them are generally predefined, instead, they are found in the personal profile database. The proper operation of the system greatly depends on the applied rule base, which should be patient-specific as far as possible [21]. For this reason, the rule base is formed based on the above factors, and the current risk level is calculated in real-time by evaluating the measured physiological parameters. In the system model structure 
the factors are classified based on their nature. The subsystems are formed based on the clusters which belong to different risk groups and they are operated as independent systems, thus the risk assessment framework can be applied for them separately. The model structure is shown in Fig. 1.

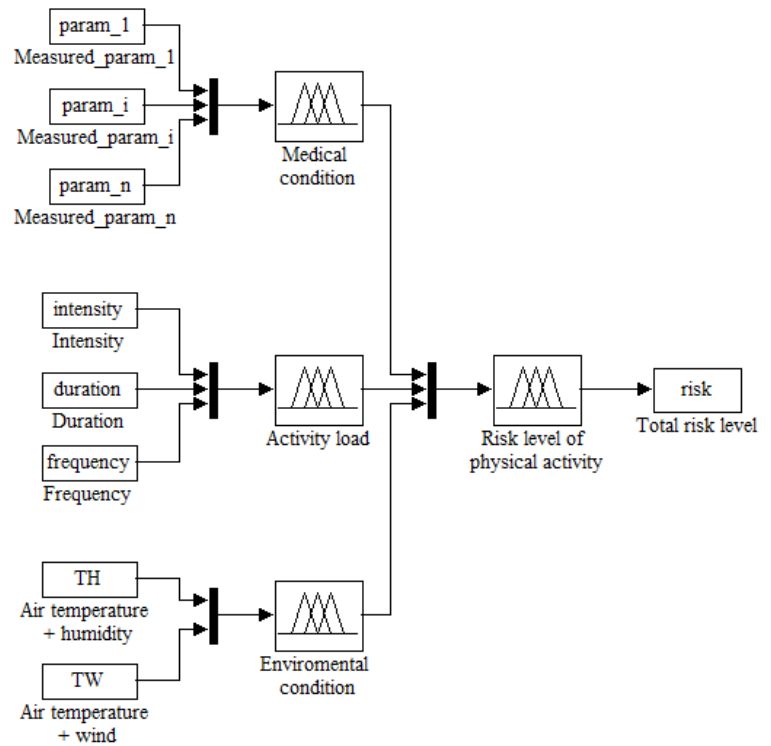

Figure 1

The model structue

The risk assessment framework is used in the first subsystem, which is used to characterize the medical state of the patient and it is called "Medical Condition". Due to the patient-specific design of the database, the personal characteristics, habits and medical recommendations are built into the system in the rule base and in the membership function design. Consequently, in this group the chronic diseases of the patient, diabetes, cardiovascular diseases, hypertension among others, are taken into account. The basic personal characteristics are also collected in the personal profile, therefore, they are also taken into consideration in this main group. The patient can be identified based on the user ID, which is the social security number (TAJ number in Hungary) of the patient and the personal rule base, the used parameters and the antecedent sets definition is determined by the personal profile in the database. Consequently, general inputs cannot be defined for this subsystem, they can be varied based on the personal profile defined in the database patient-specifically depending on the above factors. After this initialization the real-time parameters, which are assigned to the user, are used to assess the current state of the patient. These parameters are monitored in real-time to control the person continuously. 
The second main subsystem is the group "Activity load", which summarizes the characteristics of the patient's sport activity itself. It describes how intensively (intensity), how long per occasion (duration) and how many times per week (frequency) the patient does this activity.

The last main subsystem is the group "Environmental Condition", which is mainly important for outdoor sports. It combines the air temperature with wind (TW) and humidity $(\mathrm{TH})$, these form the two input parameters of this group.

The system's structure allows the expansion and modification of the inference method, which can be performed easily in each subsystem separately, thus the proposed framework can also be applied for each subsystem and organized hierarchically. Since the risk assessment framework is based on a personal database, the evaluation can be updated according to the patient's current health state (e.g. novel personal characteristics, change of the limits) and the current facilities (e.g. novel measuring devices). The modification can be performed by the expert without any programming skills through a user-friendly application.

\subsection{Database Design Providing the Flexibility of the Framework}

To ensure the appropriate operation of the system, a properly designed database structure is necessary [22], which contains the personal information; the parameters to tune the membership functions; and important information on the conditions.

During the design process it is essential to keep in mind certain principles to obtain an appropriate data model. These are the following [23], [24]:

1 Realistic modeling: the plan must correspond exactly with the specification, i.e. the entity sets, their attributes and the relationships between them should be meaningful.

2 Avoid redundancy: the multiple occurrences has a greater storage requirement, furthermore it can cause modification anomalies, therefore, it should be avoided in the system.

3 Simplicity: Only as many items should be included in the database plan as are really needed.

4 Choosing the right item: During the planning it should be decided whether an item is used as a simply implemented attribute, or as an entity set.

A database structure was designed and implemented, which is used behind the risk assessment framework and it is based on the above requirements as demonstrated in [25]. Usually the first step of the design is to create an entity-relationship (E/R) diagram (keeping in mind the above-described principles in Section 5.1), which provides a graphical representation of data modeling for ease of transparency. The obtained E/R diagram structure is shown in Fig. 2. 


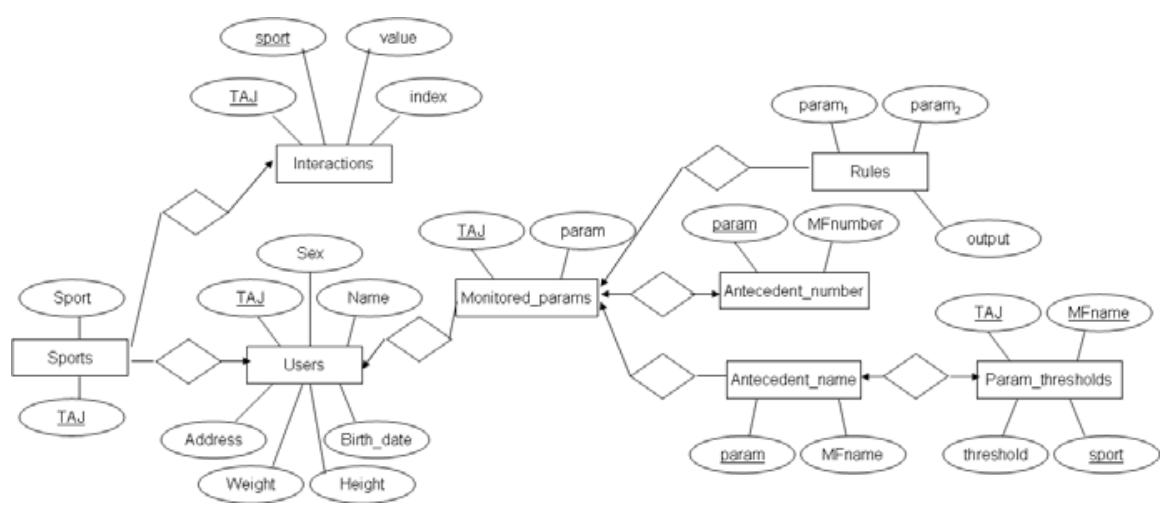

Figure 2

The entity-relationship diagram of the sport activity risk calculation framework's database ${ }^{1}$

Using the data in the model the membership functions can be tuned based on the personal characteristics, and medical recommendations. It can specify the necessary parameters for each user, which should be taken into account during the evaluation, and what types of activities are expected during the monitoring. In the database the number and name of the antecedent sets belonging to each input is given independently from the user, but the input membership function parameters can be adjusted in a patient-specific way. Also as a part of the personal profile, the risk factors' personalized relationship to one another can be defined. The parameters are arranged in pairs and an importance factor can be assigned to them as in the method AHP, whose detailed description can be seen in Section 3 . During the rule definition the personal characteristics and medical recommendations can also be taken into account by the personalized rule base, which can be stored for each user separately.

The input parameter set of the system can be expanded dynamically. In this case, the properties of the new factor should be described (the number of the input membership functions and the name of the antecedent sets). The necessary parameters can also be changed per user and the existing parameters can be combined with each other freely according to the personal endowments. Due to the above changes the rule base and the interaction between the input parameters also vary, but the relations can be easily expanded and updated. The expansion,

1 A : Entity sets: A group of individuals with similar characteristics, it is similar to the class concept, which is used in object-oriented programming languages.

$\bigcirc$ : Attributes: it serves to describe the properties of an individual.

: Relationships: two or more individuals are connected by them. These relationships are bi-directional and they can connect several entity sets. The connection types can be marked using arrows.

Underlined attribute name: Keys: these kinds of attributes or these kinds of attributes' sets, which can clearly identify an entity in the entity set. 
the parameter combination, the associated rule base and the comparison matrix can be defined by the expert without programming tasks.

Before the realization of the model, it should be translated into a relational model, which was introduced in the 1970s by Codd [20], but even today it is one of the most effective solutions. Consequently, most of the database management systems are relational [23], in which the data are represented in 2-dimensional tables, in so-called relations, in whose header there are the attribute names that define the meanings of the data in their column (relation(attributes)). The queries can be expressed in a high-level language, which significantly increases the efficiency of the database programming [26]. Precise knowledge of the database structure is essential to obtain a well-tuned system therefore the detailed description of the relations is given in the following section at the evaluation steps, where they are used.

\section{The Evaluation Process}

In this section the preparation and the steps of the evaluation are presented. Before the first-time application of the algorithm the database should be initialized. Otherwise, if any change occurs in the patient's condition (e.g. novel personal characteristics, change of the limits), or in the current facilities (e.g. novel measuring devices) then it should be updated. Failing that, the proper operation of the system cannot be guaranteed.

\subsection{Database Initialization}

The basic relations, which are applied during the evaluation, are defined in the following basic relations.

Users(TAJ, Name, Address, Birth_date, Sex, Height, Weight). This relation contains basic information of the user and they can be identified based on these values. The key attribute of this table is the $T A J$, which is the social security number of the patient.

Sports(TAJ, sport). This table contains the possible motion forms, which the person usually performs, i.e. during which the monitoring is expected. The key attribute is $T A J$.

Antecedent_number(param, MFnumber). Within the input domain different ranges belong to each measured parameter. These ranges form the antecedent sets, which are used in the fuzzy inference system and the number of them depends on the measured parameter characteristics. For example if these ranges define the normal, increased or abnormal response of the measured value then the number of the ranges are three, but is not the same for all the monitored parameters. This relation 
is used to define the number of the antecedent sets for each possible parameter. This value does not depend on the patient, it is parameter-specific, therefore the key attribute of this relation is param.

Antecedent_name(param, MFname). This relation is closely linked to the relation Antecedent_number, because it is used to define the names belonging to each antecedent set. Therefore, the number of the different antecedent name is equivalent to the number of the antecedent sets, which is defined in the relation Antecedent_number. These names do not depend on the patient, they are parameter-specific, thus here the key attribute is param.

\subsection{Determination of the Relative Importance of the Factors}

Since the input parameters of the system are not equally important during the evaluation process, a need arises for a method, which ensures their weighting. For this purpose the Analytic Hierarchy Process (AHP) is used, which is a hierarchical decision method, which was introduced by Thomas L. Saaty in the 1970s [27]. It is used for organizing and analyzing complex decisions based on a pairwise comparison matrix, which contains the relative importance of all the possible pairs of the examined factors, which are at the same level of the hierarchy. It provides an opportunity to describe both qualitative and quantitative parameters. Let the elements of the $A_{n \times n}$ comparison matrix be $X_{1}, X_{2}, \ldots, X_{n}$, where $a_{i j}$ is the relative importance of the elements $X_{i}$ and $X_{j}$. Saaty suggested values between 1 and 9 [28], but other values can also be used [29]. If $a_{i j}>1$ then $X_{i}$ is more important than $X_{j}$, consequently the elements in the main diagonal $a_{i i}=1$ and $a_{j i}=1 / a_{i j}(i=1,2, \ldots, n$; $j=1,2, \ldots n)$, therefore $n(n-1) / 2$ comparisons are required for all $n$-element groups in each level of the hierarchy. If such groups can be formed from the factors, which are more or less independent, then the comparison matrix has a block diagonal matrix form and the computational complexity can be reduced in this way. The blocks are the comparison matrices of the factors of each group and the elements in their main diagonal are 1 because in this case the factor is compared to itself [30].

Let $x=\left(x_{1}, x_{2} \ldots x_{n}\right)$ be the input vector, which is the realisation of the actual risk factors $X=\left(X_{1}, X_{2} \ldots X_{n}\right)$, then the influence of the pairwise comparison matrix $(A)$ on the actual factors can be described by the transformation $A \cdot x^{T}$. The aim is to calculate the weights of the factors, and the weighted input vector is to be forwarded to the system. The weight-multiplier $\lambda$ obtained from the pairwise comparison by $A x^{T}=\lambda x^{T}$.

The $\lambda$ values can be computed as the real eigenvalues of the comparison matrices as if they exist, otherwise the value $\lambda=1$ is used. If more than one appropriate result can be obtained then the eigenvalue should be chosen, which keeps the input vector in the universe, while it has the most influence on the decision, i.e. which has the highest efficiency. 
The above described method should be applied before the decision making process, the weighted input vectors are the system inputs [30], [31]. Due to the particularity of the task, it is a useful tool in structured risk assessment models, such as the authors' risk assessment framework. All of the risk factors and risk levels can be quantitative or given in linguistic form. The rules in the system are described in IF condition THEN consequent form. The evaluation of the risk factors and the determination of the decision is obtained by an approximate inference procedure (e.g. Mamdani-type inference system, Mamdani-like structure with discretized output [32]). The system can operate with or without feedback, in the latter case it determines the risk level or it makes a decision depending on the task's nature [33].

\subsection{The Steps of the Evaluation}

In the following algorithm the steps of the evaluation for a subsystem in the case of the Mamdani-like structure with discretized output detailing the database relations can be seen, which are used in each step to personalize the decision making. If a multilevel, clustered system structure is used then this algorithm should be executed for each subsystem separately.

Step 1 The input parameter combination is not constant, the number of the inputs and the monitored parameters can vary patient- and sportspecifically. The current combination is defined in the personal profile in the relation Monitored param( $\underline{T A J}$, sport, param) according to the health state of the patient and the sport type should also be considered. Consequently, the key attributes are $T A J$ and sport together.

The so-defined input values are fuzzified in the case of trapezoidal membership functions by (1)

$$
\mu_{A}(x)=\left\{\begin{array}{cc}
0 & x \leq a_{i} \\
\frac{x-a_{i}}{b_{i}-a_{i}} & a_{i} \leq x \leq b_{i} \\
1 & b_{i} \leq x \leq c_{i} \\
\frac{d_{i}-x}{d_{i}-c_{i}} & c_{i} \leq x \leq d_{i} \\
0 & d_{i} \leq x
\end{array}\right.
$$

where $a_{i}, b_{i}, c_{i}, d_{i}$, are the parameter sets, which can be used to tune the membership functions patient-specifically based on the values defined in the framework's database according to the medical recommendations in

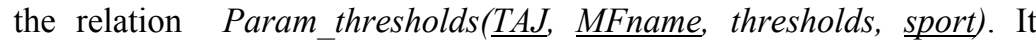
should be defined for each parameter, which are used to characterize the patient, i.e. which are defined in the table Monitored_param. These values define the minimum and maximum values of each range, which 
are assigned to each antecedent set. The parameters of the antecedent sets can be obtained from these values, which, apart from the antecedent set, are strongly related to the patient, the type of the monitored sport activity, consequently the key attributes are TAJ, MFname and sport together.

Step 2 The definition of the rule premises $\left(w_{i}\right)$ by (2) from the fuzzified values, which are weighted by the values obtained from the AHP method, whose results are stored in the relation Interactions( $\underline{T A J}$, sport, index, value). This relation defines the relative importance of the parameters in a patient-specific way depending on the type of sport. A default comparison matrix can be defined for each parameter combination, but it can be modified according to the patient's personal characteristics and chronic diseases.

The weighting of the input factors is not ensured in the Mamdani inference based systems, when the product operator is used during the determination of the rule premises. The product operator conceals weighting due to its commutative property, i.e. if different weights are used in different order, so they are assigned to another parameter, the result does not change. Consequently, the basic algorithm should be modified to take into account the different weighting factors.

$$
w_{i}=\left\{\begin{array}{cc}
\sum_{k=1}^{m} a_{k} \mu_{A_{k j}}\left(x_{k}\right) & \text { if } \\
0 & \text { otherwise }
\end{array} \quad \mu_{A_{k j}} \neq 0, \forall k \in[1, m]\right.
$$

where $m$ is the number of the input parameters, $a_{k}$ is the weight belonging to input $k, w_{i}$ is the firing strength of the rule premise $i$.

Step 3 The firing strength obtained in the previous step should be normalized by (3) to ensure the appropriate operation of the system.

$$
\bar{w}_{i}=\frac{w_{i}}{\sum_{i=1}^{n} w_{i}}
$$

Step 4 The defuzzification of each consequent part happens separately using COG method (4), trapezoidal membership functions can be used again. The consequent parts $\left(\mu_{D_{i}}(y)\right)$ belonging to each rule-premise are

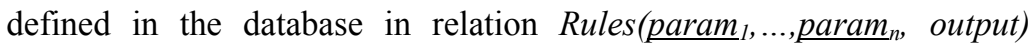
patient- and sport-specifically. The key attributes of this relation are the parameter combination, which define the rule-premise in step 2. 


$$
f_{i}=\frac{\int_{\operatorname{supp}_{D_{i}}} \mu_{D_{i}}(y) y d y}{\int_{\operatorname{supp}_{D_{i}}} \mu_{D_{i}}(y) d y}
$$

Step 5 The final step of the evaluation is the overall risk level $(O)$ calculation of the subsystem by (5).

$$
O=\sum_{i=1}^{n} \bar{w}_{i} f_{i}=\frac{\sum_{i=1}^{n} w_{i} f_{i}}{\sum_{i=1}^{n} w_{i}}
$$

\section{The Risk Assessment Framework Implementation}

\subsection{Test Environment}

The risk assessment framework has been implemented and its performance has been tested in RAD Studio application development environment. This development tool integrates Delphi, C++ Builder and HTML5 Builder, thus allows the broad applicability of the software for multiple devices (PCs, tablets, smart phones) on multiple platforms (iOS, Windows, Mac) in a native form [34]. The testing was performed on a PC using Windows platform. The hardware environment, used to test the model, is Intel ${ }^{\circledR}$ Core $^{\mathrm{TM}} 2$ Duo CPU T6570 processor with 4GB RAM and Windows7 operating system. The input data are stored in a structured text file, which is based on medical databases.

\subsection{The Default Values in the Database}

In the case, when there is no detailed personal profile with specified medical recommendations, the program is able to calculate the thresholds of the measured parameters and these thresholds can be used as default values in the database before the personalization. The expert can modify them if it is necessary, but they can be retained, if they characterize the patient properly. In the following section some examples are presented for the measured parameters' default threshold calculation. 


\subsubsection{The Systolic Blood Pressure (SBP)}

A formula (6) was defined to calculate the thresholds of the systolic blood pressure responses for patients. This equation serves as a basis of the membership function parameter calculation.

$$
S B P_{\text {limit }}(\text { target })=\left(S B P_{\text {limit }}^{\text {normal }}-S B P_{\text {rest }}\right) \cdot \text { zone }+S B P_{\text {rest }}
$$

where zone is the center of the recommended zone, limit is the range limit, which can be maximum or minimum. The values of $\mathrm{SBP}_{\text {limit }}^{\text {normal }}$ are based on the values defined by Sieira et al. (see Table 1), which are the thresholds of normal systolic blood pressure responses of both sexes with ages between 20 and 79 [11].

Table 1

Normal Systolic Blood Pressure (SBP, [Hgmm]) response

\begin{tabular}{|c|c|c|c|c|}
\hline Age & \multicolumn{2}{|c|}{ Men } & \multicolumn{2}{c|}{ Women } \\
\hline & $S B P_{\min }^{\text {normal }}$ & $S B P_{\max }^{\text {normal }}$ & $S B P_{\min }^{\text {normal }}$ & $S B P_{\max }^{\text {normal }}$ \\
\hline $20-29$ & 161 & 203 & 136 & 176 \\
\hline $30-39$ & 164 & 204 & 138 & 182 \\
\hline $40-49$ & 167 & 209 & 144 & 190 \\
\hline $50-59$ & 170 & 216 & 153 & 201 \\
\hline $60-69$ & 173 & 221 & 162 & 210 \\
\hline $70-79$ & 169 & 223 & 160 & 210 \\
\hline
\end{tabular}

\subsubsection{The Heart Rate (HR)}

The default heart rate thresholds can be obtained from the predictive maximum heart rate of the patient calculated by Polar training computer [35]. The zones defined by the thresholds can be obtained as a percentage of this value based on the health condition or physical fitness of the patient, which basically determines the allowable intensity of the activity. The zones, which are used in the author's framework, are the modification of the Polar target zones [12], [36], and they are defined in Table 2.

Table 2

Physical fitness and health state zones

\begin{tabular}{|c|l|}
\hline $\begin{array}{c}\text { Intensity \% of } \\
\mathbf{H R}_{\text {max }} \mathbf{( b p m )}\end{array}$ & \multicolumn{1}{c|}{ Recommended for patients who } \\
\hline$<50 \%$ & $\begin{array}{l}\text { are under: medical treatment/rehabilitation } \\
\text { suffer from cardiac disease }\end{array}$ \\
\hline $50-70 \%$ & $\begin{array}{l}\text { exercise as prevention } \\
\text { are beginners } \\
\text { do basic training }\end{array}$ \\
\hline
\end{tabular}




\begin{tabular}{|c|l|}
\hline $\begin{array}{c}\text { Intensity \% of } \\
\mathbf{H R}_{\mathbf{m a x}}(\mathbf{b p m})\end{array}$ & \multicolumn{1}{|c|}{ Recommended for patients who } \\
\hline$<50 \%$ & $\begin{array}{l}\text { are under: medical treatment/rehabilitation } \\
\text { suffer from cardiac disease }\end{array}$ \\
\hline $70-95 \%$ & do sports regularly \\
\hline
\end{tabular}

\subsection{Case Study}

In this section a 28 years old male is considered to illustrate the usage of the framework. The relation Sports contains the possible motion form for this user.

Table 3

Relation Sports

\begin{tabular}{|c|c|}
\hline TAJ & Sport \\
\hline 024357942 & running \\
\hline 024357942 & Cycling \\
\hline
\end{tabular}

The relation Monitored_param (Table 4) defines the input parameter of the Medical condition subsystem in the framework. If the patient uses the system during cycling then their heart rate (HR), systolic blood pressure (SBP), and diastolic blood pressure (DBP) should be measured and evaluated; during running heart rate and respiration rate $(\mathrm{RR})$ are considered.

Table 4

Relation Monitored_param

\begin{tabular}{|c|c|c|}
\hline TAJ & Sport & Param \\
\hline 024357942 & running & (HR, RR) \\
\hline 024357942 & cycling & (HR, SBP, DBP) \\
\hline
\end{tabular}

The number of the antecedent sets assigned to each parameter is stored in the relation Antecedent_number (Table 5) and the names of these sets are defined by relation Antecedent_name (Table 6). These values are parameter-independent, therefore they are assigned to each parameter generally.

Table 5

Relation Antecedent_number

\begin{tabular}{|c|c|}
\hline Param & MFnumber \\
\hline HR & 3 \\
\hline SBP & 4 \\
\hline DBP & 4 \\
\hline RR & 3 \\
\hline
\end{tabular}


Table 6

Relation Antecedent_name

\begin{tabular}{|c|c|}
\hline Param & MFname \\
\hline HR & (target, mhigh, vhigh) \\
\hline SBP & (low, normal, increased, abnormal) \\
\hline DBP & (lower, average, arisen, irregular) \\
\hline RR & (normal, higher, RRvhigh) \\
\hline
\end{tabular}

In order to obtain patient-specifically tuned membership functions the relation Param_thresholds (Table 7) should be used supplementing the above membership function information.

Table 7

Relation Param_thresholds

\begin{tabular}{|c|c|c|c|}
\hline TAJ & MFname & Threshold & Sport \\
\hline 024357942 & Target & 0.7 & Cycling \\
\hline 024357942 & Mhigh & 0.85 & Cycling \\
\hline 024357942 & Low & 125 & Cycling \\
\hline 024357942 & Normal & 165 & Cycling \\
\hline 024357942 & Increased & 190 & Cycling \\
\hline
\end{tabular}

Using the obtained data in the case of the 28 -year-old male patient, whose $\mathrm{HR}_{\max }$ is $197 \mathrm{bpm}, \mathrm{HR}_{\text {rest }}$ is $70 \mathrm{bmp}$, and he exercises as prevention, the following input membership functions are created.

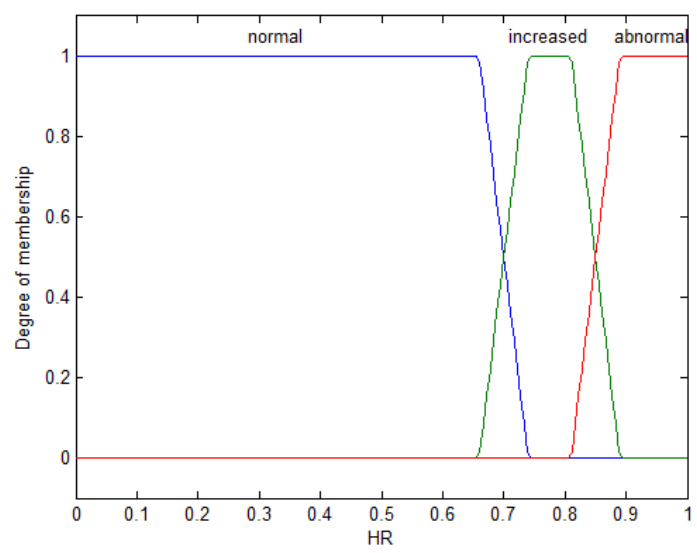

Figure 3

Heart rate membership functions for the 28-year-old male 


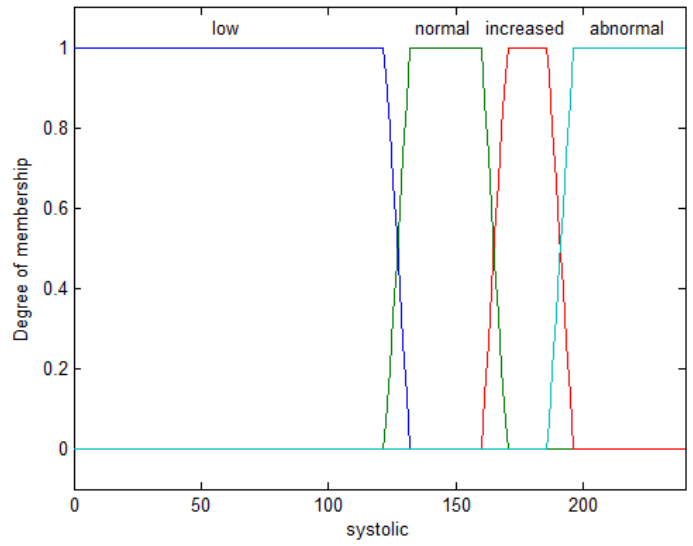

Figure 4

Systolic blood pressure membership functions for the 28-year-old male

There are some examples of obtained risk levels for different input values, which are presented in Table 8 . The risk levels are considered in [0,1] interval.

Table 8

Calculated risk levels

\begin{tabular}{|c|c|c|c|}
\hline HR & Systolic & Diastolic & Risk level \\
\hline 130 & 140 & 70 & 0.16 \\
\hline 130 & 180 & 90 & 0.65 \\
\hline 170 & 180 & 90 & 0.79 \\
\hline 140 & 150 & 75 & 0.26 \\
\hline
\end{tabular}

\subsection{The Test Results}

The basis of the testing method an AHP-FCE method was [37] to compare the results obtained from weighted input factors. For the sake of comparability, the evaluation was performed for the same patients, utilizing the same input values and the same membership functions in both systems. This condition did not allow the exploitation of the novel model's benefits, but it showed that the results obtained from the comparison are the same. Consequently, the risk assessment framework by Tóth-Laufer, Takács and Rudas was validated.

However, the importance of the presented novel framework lies in the fact that it makes the evaluation more differentiable. Using the appropriate settings the patients with the apparently same parameters can be evaluated differently, over and above the different parameter combinations can be assigned to different sport types. In this way the result is more realistic and patient-specific. In Table 9, the results obtained by using different membership function parameters are shown to illustrate the change in the risk level according to them. These boundaries are 
defined in the relation Parameter_thresholds according to the medical recommendations, all other inputs are the same as in the case study. The obtained results highlighted that for the more specified values the result is shifted according to the patient's characteristics.

Table 9

Risk levels (Inputs: HR=130 bpm, $\mathrm{SBP}=180 \mathrm{Hgmm}, \mathrm{DBP}=90 \mathrm{Hgmm}$ )

\begin{tabular}{|c|c|c|c|c|c|}
\hline Target & Mhigh & Low & Normal & Increased & Risk \\
\hline 0.7 & 0.85 & 135 & 165 & 190 & 0.65 \\
\hline 0.5 & 0.6 & 125 & 149 & 155 & 0.9 \\
\hline 0.6 & 0.7 & 130 & 160 & 170 & 0.89 \\
\hline 0.65 & 0.75 & 140 & 165 & 180 & 0.79 \\
\hline 0.75 & 0.9 & 138 & 175 & 198 & 0.7 \\
\hline
\end{tabular}

\section{Conclusions}

In health monitoring systems, the appropriate evaluation process, which provides realistic result, is a highly problematic task. It is the consequence of the high number of input factors, which includes both quantitative and qualitative parameters; the different importance of them; the complex interactions between them; and consequently, the large rule base. Furthermore, for different patients different parameter combinations should be considered and the thresholds of normal, increased or abnormal values cannot be generalized, they should be adjusted is a patient-specific way.

Some of the above problems can be handled by the fuzzy approach, which is able to work with uncertainty, imprecision and subjectivity in the data and in the evaluation process. The large rule base can also be reduced by different fuzzybased techniques and the membership functions can be tuned according to the patient characteristics, but these kinds of systems are not flexible enough. The parameter combinations cannot be varied, and broadened for each user separately.

A risk assessment framework was designed and implemented, which takes the advantages of the fuzzy approach, while the flexibility, expandability and the adaptive capacity of the system is improved. To ensure the above advantages and to achieve transparency a general, modular system structure is used, which is based on the specifically configurable subsystems. During the evaluation the individual characteristics, living conditions, habits and medical recommendations are considered, which are based on the personal profile, in which different parameter combinations can be defined for each user and these can be modified and broadened user-specifically. In this way the obtained result is more realistic. The results of the proposed model was compared to an AHP-FCE model for the same input values. The results obtained from the comparison are the same, consequently the authors' risk assessment framework was validated. 


\section{Acknowledgement}

The research was supported by the Hungarian OTKA projects 106392 and 105846, and project of the Vojvodina Academy of Sciences and Arts "Mathematical models of intelligent systems and theirs applications".

\section{References}

[1] L. Hanka, "Kockázat becslése numerikus módszerekkel, folytonos eloszlások diszkretizálása”, Müszaki Katonai Közlöny Online XXII, No. 3, 2012, pp. 55-69

[2] M. Takács, "Multilevel Fuzzy Approach to the Risk and Disaster Management", Acta Polytechnica Hungarica, Vol. 7, No. 4, 2010, pp. 91102

[3] E. Tóth-Laufer, et al., "Current Physical Status Evaluation Subsystem using User-specific tuned Membership Functions in Sport Activity Risk Calculation" in Proc. of the IEEE $9^{\text {th }}$ International Conference on Computational Cybernetics, Tihany, Hungary, July 8-10, 2013, pp. 185190, ISBN: 978-1-4799-0060-2, DOI: 10.1109/ICCCyb.2013.6617585

[4] M. Kozlovszky, J. Sicz-Mesziár, et.al., "Combined Health Monitoring and Emergency Management through Android Based Mobile Device for Elderly People," Wireless Mobile Communication and Healthcare, Lecture Notes of the Institute for Computer Sciences, Social Informatics and Telecommunications Engineering, Vol. 83, 2012, pp. 268-364

[5] B. Najafi, K. Aminian, "Measurement of Stand-Sit and Sit-Stand Transitions Using a Miniature Gyroscope and its Application in Fall Risk Evaluation in the Elderly," IEEE Trans. on Biomedical Engineering, Vol. 49, No. 8, Aug. 2002, pp. 843-351, doi: 10.1109/TBME.2002.800763

[6] S. Dang, S. Dimmick, G. Kelkar, "Evaluating the Evidence Base for the Use of Home Telehealth Remote Monitoring in Elderly with Heart Failure", Telemedicine and e-Health, October 2009, Vol. 15, No. 8, pp. 783-796, doi:10.1089/tmj.2009.0028

[7] M. Pallikonda Rajasekaran, et al., "Elderly Patient Monitoring System Using a Wireless Sensor Network", Telemedicine and e-Health, January 2009, Vol. 15, No. 1, pp. 73-79, doi:10.1089/tmj.2008.0056

[8] M. Kozlovszky, Z. Meixner, et al. "Network and Service Management and Diagnostics Solution of a Remote Patient Monitoring System", in Proc. of the $3^{\text {rd }}$ IEEE International Symposium on Logistics and Industrial Informatics, Budapest, Hungary, August 25-27, 2011, pp. 61-64, doi: 10.1109/LINDI.2011.6031163

[9] J. Min Kang, T. Yoo, H.Chan Kim, "A Wrist-Worn Integrated Health Monitoring Instrument with Tele-Reporting Device for Telemedicine and Telecare", IEEE Transactions on Instrumentation and Measurement, Vol. 55, No. 5, October 2006, pp. 1655-1661, doi: 10.1109/TIM.2006.881035 
[10] B. Meade, "Emergency Care in a Remote Area using Interactive Video Technology: A Study in Prehospital Telemedicine", J. Telemed. Telecare, Vol. 8, No. 2, Apr. 2002, pp. 115-117

[11] M. C. Sieira, A. O. Ricart, R. S. Estrani, "Blood Pressure Response to Exercise Testing“ in Apunts Med Esport., Elsevier, 2010, pp. 191-200

[12] K. Hottenrott, "Training with the Heart Rate Monitor", Meyer and Meyer Verlag, 2007, ISBN: 9781841262130

[13] F. Rahnman, et al., "Network Approach for Physiological Parameters Measurement," IEEE Trans. on Instrumentation and Measurement, Vol. 54, No. 1, Feb. 2005, pp. 337-346, doi: 10.1109/TIM.2004.834595

[14] E. Tóth-Laufer, A. R. Várkonyi-Kóczy, “A Soft Computing-based Hierarchical Sport Activity Risk Level Calculation Model for Supporting Home Exercises", IEEE Trans. on Instrumentation and Measurement, Vol. 63, No. 6, 2014, pp. 1400-1411, DOI: 10.1109/TIM.2014.2299523

[15] Y. Koyama, M. Nishiyama, K. Watanbe, "A Motion Monitor Using HeteroCore Optical Fiber Sensors Sewed in Sportswear to Trace Trunk Motion," IEEE Trans. on Instrumentation and Measurement, Vol. 62, No. 4, Apr. 2013, pp. 828-836, doi: 10.1109/TIM.2013.2241534

[16] G. Hache, E. D. Lemaire, N. Baddour, "Wearable Mobility Monitoring Using a Multimedia Smartphone Platform", IEEE Trans. on Instrumentation and Measurement, Vol. 60, No. 9, Sept. 2011, pp. 31533161, doi: 10.1109/TIM.2011.2122490

[17] A. Karime, M. Eid, J. M. Alja'am, A. E. Saddik, "A Fuzzy-based Adaptive Rehabilitation Framework for Home-Based Wrist Training", IEEE Trans. on Instrumentation and Measurement, Vol. 63, No. 1, 2013, pp. 135-144, doi: 10.1109/TIM.2013.2277536

[18] A. Alamri, Cha Jongeun, A. El Saddik, "AR-REHAB : An Augmented Reality Framework for Poststroke-Patient Rehabilitation", IEEE Trans. on Instrumentation and Measurement, Vol. 59, No. 10, Oct. 2010, pp. 25542563, doi: 10.1109/TIM.2010.2057750

[19] G. Fördős, I. Bosznai, et al., "Sensor-net for Monitoring Vital Parameters Vehicle Drivers", Acta Polytechnica Hungarica, Vol. 4, No. 4, 2007, pp. 25-36

[20] E. F. Codd, „A Relational Model for Large Shared Data Banks”, Comm. $A C M, 136$, pp. $377-387$

[21] Zs. Cs. Johanyák, O. Papp, “A Hybrid Algorithm for Parameter Tuning in Fuzzy Model Identification”, Acta Polytechnica Hungarica, Vol. 9, No. 6, 2012, pp. 153-165 
[22] A. Keszthelyi, „The Role of Data modelling in Information System Efficiency", in Teaching and learning, $2^{\text {nd }}$ International Conference for Theory and Practice in Education, 29 May 2009, Budapest, p. 26

[23] J. D. Ullman, J. Widom, „A First Course in Database Systems”, PrenticeHall, 2007

[24] S. Sergyán, L. Csink, "Consistency Check of Image Databases", in Proc. of the $2^{\text {nd }}$ Romanian-Hungarian Joint Symposium on Applied Computational Intelligence, Timisoara, Romania, May. 12-14, 2005, pp. 201-206

[25] E. Tóth-Laufer, "Database Schema Design for Supporting Sport Activity Monitoring," in Proc. of the $18^{\text {th }}$ International Conference on Intelligent Engineering Systems, Tihany, Hungary, July 3-5, 2014, pp. 143-149

[26] A. Keszthelyi, "Remarks on the Efficiency of Information System", in Acta Polytechnica Hungarica, Vol. 7, No. 3, 2010, pp. 153-161

[27] T. L. Saaty, "The Analytic Hierarchy Process: Planning, Priority Setting, Resource Allocation", McGraw-Hill, 1980, ISBN 0-07-054371-2

[28] T. L. Saaty, L. G. Vargas, "Models, Methods, Concepts and Applications of the Analytic Hierarchy Process", Kluwer Academic Press, 2001

[29] L. Mikhailov, "Deriving Priorities from Fuzzy Pairwise Comparison Judgements", Fuzzy Sets and Systems, Vol. 134, 2003, pp. 365-385

[30] M. Takács, E. Tóth-Laufer, "The AHP Extended Fuzzy-based Risk Management" in Proc. of the $10^{\text {th }}$ WSEAS Int. Conference on Artificial Intelligence, Knowledge Engineering and Data Bases, Cambridge, UK, February 20-22, 2011, pp. 269-272, ISBN: 978-960-474-273-8

[31] T. L. Saaty, "Decision Making with the Analytic Hierarchy Process", Int. J. Services Sciences, Vol. 1, No. 1, 2008, pp. 83-98

[32] E. Tóth-Laufer, I. J. Rudas, M. Takács, „Operator Dependent Variations of the Mamdani-type Inference System Model to Reduce the Computational Needs in Real-Time Evaluation“, International Journal of Fuzzy Systems, Vol. 16, No. 1, March 2014, pp. 57-72

[33] J. H. M. Carr, V. Tah, "A Fuzzy Approach to Construction Project Risk Assessment and Analysis: Construction Project Risk Management System", Advances in Engineering Software, Vol. 32, No. 10, October, 2001, pp. $847-857$

[34] RAD Studio - Embarcadero Technologies Product Documentation, http://docs.embarcadero.com/products/rad_studio/

[35] Polar RS100 User Manual, http://www.polarusa.com/support_files/usen/85256F470048B0BC852574730060F559/Polar_RS100_user_manual_E nglish.pdf 
[36] Polar RS800CX User Manual, Polar USA, http://www.polar.com/ e_manuals/RS800CX/Polar_RS800CX_user_manual_English/manual.pdf

[37] Y. Wu, Y. Ding, H. Xu, "Comprehensive Fuzzy Evaluation Model for Body Physical Exercise" in Risk Life System Modeling and Simulation Lecture Notes in Computer Science, 2007, Vol. 4689/2007, pp. 227-235, doi: 10.1007/978-3-540-74771-0_26 